\title{
A Prospective Real-World Study Exploring Associations Between Passively Collected Tracker Data and Headache Burden Among Individuals with Tension-Type Headache and Migraine
}

\author{
Christian J. Cerrada · Jae S. Min · Luminita Constantin • \\ Simon Hitier · Iva Igracki Turudic · Caroline Amand-Bourdon • \\ Andrew Stewart · Caty Ebel-Bitoun · Peter J. Goadsby
}

Received: August 12, 2021 / Accepted: October 22, 2021 / Published online: December 7, 2021

(C) The Author(s) 2021

\section{ABSTRACT}

Introduction: Prevalence and burden of headache disorders in real-world settings is relatively unstudied. We explored the associations between passively collected activity data, headache burden, and quality of life in headache sufferers.

Supplementary Information The online version contains supplementary material available at https:// doi.org/10.1007/s40122-021-00336-y.

C. J. Cerrada $\cdot$ J. S. Min

Evidation Health, San Mateo, CA, USA

L. Constantin · S. Hitier · C. Ebel-Bitoun

Consumer Healthcare, Sanofi, Gentilly, France

I. Igracki Turudic

Consumer Healthcare, Sanofi, Frankfurt, Germany

C. Amand-Bourdon

Real World Evidence, Sanofi, Gentilly, France

A. Stewart

Real World Evidence, Sanofi, Cambridge, MA, USA

P. J. Goadsby $(\bowtie)$

NIHR-Wellcome Trust King's Clinical Research

Facility, King's College Hospital, King's College

London, Wellcome Foundation Building, London

SE5 9PJ, UK

e-mail: peter.goadsby@kcl.ac.uk

P. J. Goadsby

Department of Neurology, University of California, Los Angeles, CA, USA
Methods: Data from wearable activity tracking devices and daily short questionnaires were collected over 12 weeks to assess occurrence of headache, activity, quality of life and self-rated health. Variables were analyzed using a series of mixed-effects models and stratified based on headache type. Multiple linear and logistic regressions were used to analyze treatment preferences.

Results: Behaviors inferred from activity tracker data suggested that individuals slept more, had reduced physical activity, and had lower maximum heart rate on days with headache. As headache-specific impact on quality of life increased, activity and maximum heart rate decreased and sleep increased. Headache days with higher self-rated health were associated with less napping, higher step count and maximum heart rate, correlating with increased activity. Migraineurs experienced greater burden in everyday life compared with tensiontype headache sufferers.

Conclusion: This study adds to existing evidence that activity trackers can be used to quantify headache burden in real-world settings and aid in understanding symptom management.

Keywords: Headache; Quality of life; Trackerbased behavioral features; Real-world evidence 


\section{Key Summary Points}

This large 12-week real-world study assessed the impact of headache on selfrated health and quality of life, and also analyzed associations between headache and daily activity features derived from wearable activity tracking devices.

The analysis showed that occurrence of headache was associated with reduced daily step count, decreased proportion of the day spent active, increased daytime napping, and lower maximum heart rate.

When stratified by headache type, days with migraine were associated with increased sleep duration and decreased physical activity and maximum heart rate compared to days without migraine.

\section{INTRODUCTION}

There is a relative lack of prospective and realworld data on primary headache disorders, although recent studies indicate that stress, sleep, and daily physical activity may be linked to headache [1-3]. The widespread use of commercially available, wearable activity tracking devices presents a unique opportunity to understand daily activity among headache sufferers in an unobtrusive and less burdensome manner. This prospective, observational, exploratory study paired passively collected activity data from wearable tracking devices with online questionnaires to understand the impact of headache on daily activities and quality of life (QoL) among frequent headache sufferers, and understand how they self-manage their symptoms in real-world settings. Specifically, we explored the relationship between headache burden, i.e., occurrence, intensity levels, self-reported QoL, and treatment choices, and step count, sleep, and heart metrics from Fitbit ${ }^{\circledR}$ activity trackers (Fitbit, San Francisco, CA, USA).

\section{METHODS}

\section{Study Design}

This was a single-arm, 12-week cohort study conducted online. Potential participants were recruited through an online research study platform (Achievement Studies, Evidation Health Inc., San Mateo, CA, USA). Achievement was used to advertise, recruit, verify eligibility, obtain consent, and enroll participants into this study. This study was performed in accordance with the Helsinki Declaration of 1964 and its later amendments. Signed informed consent was obtained electronically from participants prior to enrollment using Evidation Health's eConsent process. The study protocol, informed consent form, recruitment materials, and all participant materials were approved by the Western Institutional Review Board (Puyallup, WA, USA; WIRB protocol \#20190326). Participants completed all study tasks at home on the study platform along with suitable mobile devices and Fitbit activity trackers and received compensation of up to $\$ 55$ US for their participation.

\section{Study Population}

The enrolled population was composed of headache sufferers aged $\geq 18$ years, who lived in the USA and self-reported experiencing 5-14 days with headache per month on average. Participants were required to own and encouraged to wear a Fitbit activity tracker (any model) daily for the duration of the study. Participants were excluded if they had any other pre-existing neurological condition, such as epilepsy, had a sleep disorder, such as narcolepsy or insomnia, or had a lifestyle that led to irregular or atypical circadian patterns (e.g., overnight shift work). Analysis Population 1 included all participants with daily surveys (no more than 7 consecutive days of missing surveys) and activity trackers: dense data (no more than 7 consecutive invalid days of tracker data determined by $<10 \mathrm{~h}$ of wear time based on steps data). Analysis Population 2 included all participants who had $\leq 7$ consecutive invalid days of heart rate 
data: $<10 \mathrm{~h}$ of tracker wear time determined by heart rate data stream (Fig. 1a).

\section{Study Objectives}

The primary objective was to understand the day-level association between passively collected activity data features and (1) headache occurrence on a given day (i.e., days with headache), and (2) QoL, as measured by impact on daily life and self-rated health on days with headache. The secondary objective aimed to understand headache-related treatment preferences and decisions.

\section{Data Sources and Variables}

Self-reported data were collected through baseline and daily surveys completed via email, and activity data were collected at the minute level using Fitbit activity trackers throughout the study period. At baseline, all participants completed a headache-specific QoL Migraine Disability Assessment (MIDAS) [4] and answered questions on demographics, medical history, headache type, average pain intensity, medication use, and general lifestyle. Participants also responded to questions about their headache symptoms, which were used to classify their headache types into migraine, tension-type headache (TTH), or cluster headache, based on questions specifically adapted from the International Classification of Headache Disorders 3 (ICHD-3) [5]. Daily surveys assessed the occurrence of headache and QoL on days with headache (perceived impact of headache on daily life and overall well-being [self-rated health]) each day throughout the study. Participants reported the impact of headache on daily life using an ordinal scale ranging from 1 to 5 $(1=$ no impact at all; $5=$ a great deal of impact $)$ and self-rated health (from $1=$ very poor to $5=$ very good) (Fig. 1b). Analogs of sleep, including duration, stages, disturbances, and overall sleep quality, step count, and heart rate were collected continuously throughout the study via Fitbit. At the end of the study, participants completed a final survey to assess changes in MIDAS scores and medication use.

\section{Statistical Analysis}

Results were summarized using descriptive statistics: per-participant percentage of missing data and distributional features of primary and secondary evaluation variables (median, mean, standard deviation, etc.), as deemed appropriate. All statistical analyses were performed at the 5\% significance level using two-sided tests or two-sided 95\% confidence intervals (CI); $P$ values were adjusted for the false discovery rate (FDR) via the Benjamini-Hochberg procedure. A sample size of approximately 500 participants was estimated as required to identify significant effects across activity tracker-based endpoints, accounting for FDR correction using the error rate of $5 \%$ and power of $80 \%$ and assuming that $50 \%$ of the activity features will yield an 18\% difference between days with and without headache for each feature.

In the primary objective analysis, 14 passively collected activity features related to sleep (i.e., naps, total time spent asleep, and sleep from the night before), steps (e.g., total number of steps that day), and heart rate (e.g., resting heart rate) were assessed individually as outcomes, with day with or without headache as a binary predictor, using linear and logistic mixed-effects models with random intercepts to account for intra-individual correlations. The models assessed the relationships between day with headache (with day without headache as reference comparison) and each activity feature adjusted for age and gender as covariates that were hypothesized to be associated with both activity and headache. Beta coefficients $(\beta)$ or odds ratios (ORs), 95\% CI, and q-values (FDR adjusted $P$ values) were reported for each of the models. To examine the association between sleep and headache, all sleep variables from the current night and previous night ("last night") (total hours of sleep, fraction of time spent awake, and number of times woken up) were included. In these analyses, positive associations between predictors and outcomes yield $\beta>0$ while negative relationships between predictors and outcomes yield $\beta<0 ; \beta=0$ indicates no association.

In addition to the occurrence of headache, QoL on headache days was evaluated with 
A

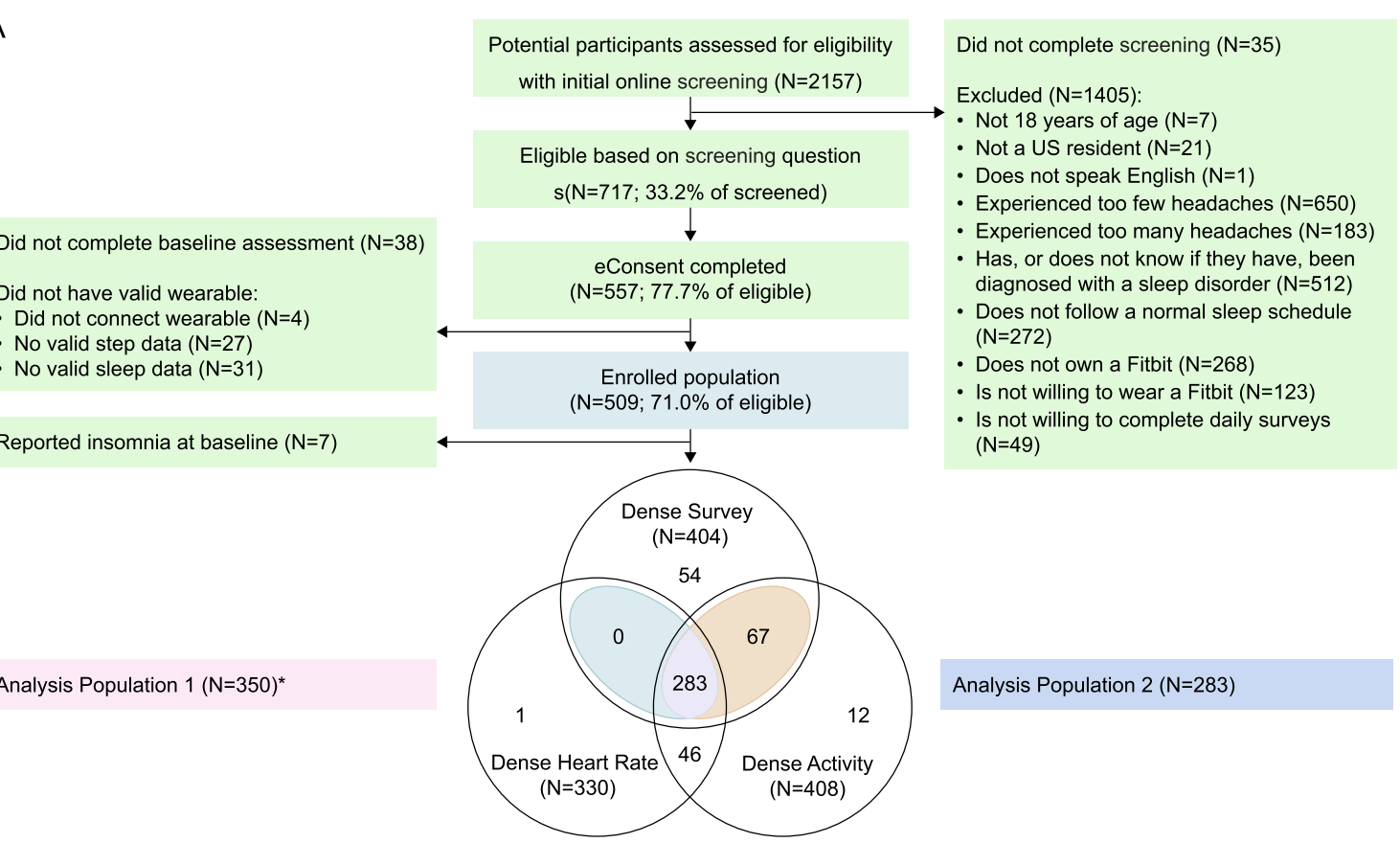

B

Behavioural patterns in individuals with headache pain

Baseline survey:

Study schedule

End-of-study survey:

- Demographics

- Medical history

- Lifestyle

- MIDAS

- Treatment patterns

Enrollment

Activity tracker data collected continuously as minute-level data

- Changes in treatment patterns

- MIDAS

Self-report surveys collected daily

- Experience with the study

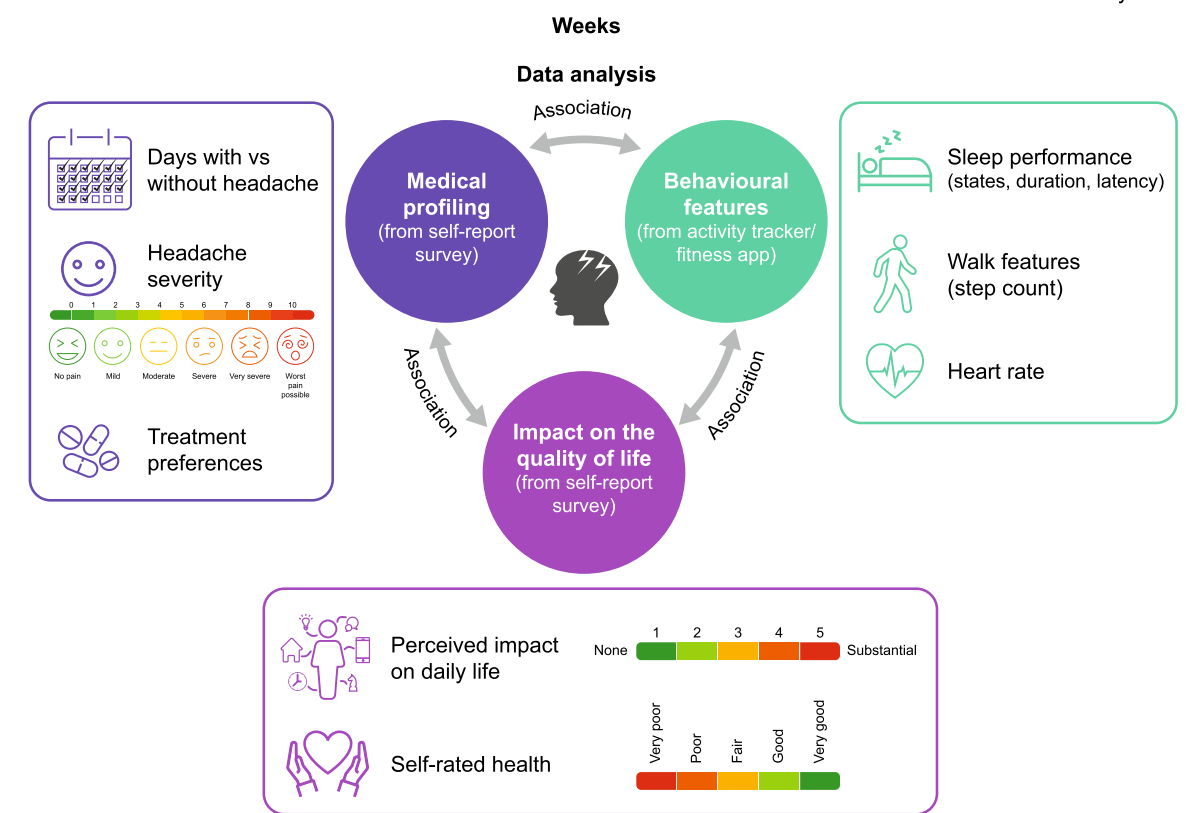


4Fig. 1 Study design (a), schedule, and data sources (b). MIDAS Migraine Disability Assessment questionnaire, $N$ number. Asterisk indictes Analysis Population 1 $(N=350)$, which included all participants who met both the dense survey criterion $(N=404)$ and the dense activity criteria $(N=408)$ by steps. Participants may have met $>1$ criterion for exclusion. Quality of life was measured using an ordinal scale ranging from 1 to 5

either headache-specific impact on daily living or self-rated health as an ordinal predictor. Similar to the mixed-effects models described above, intra-individual correlation was taken into account as random intercepts. The associations between activity features and QoL were presented as $\beta$ regression coefficients or ORs of the fixed effects, $95 \% \mathrm{CI}$, and $q$-values.

For all, the statistical significance was determined by the $q$-value (below the 0.05 threshold set a priori) or the CI (included 0 ).

For the secondary objective, descriptive summaries were generated for self-reported medication use and reasons for use based on aggregated daily survey responses over the study period. Participants were classified as prescription headache medication users based on their responses to daily surveys. Differences between groups were analyzed using multivariable linear regressions (for continuous outcomes, e.g., proportion of headache days where as-needed medication was taken) and logistic regressions (for categorical outcomes) using logit link functions, and with adjustment for multiple comparisons. Differences between prescription medicine users and non-users were examined with respect to demographics, comorbidities, headache type, pain intensity and perceived triggers, as well as activity data.

Missing data were reported and evaluated for randomness using heatmaps and exploratory analyses of associations with baseline participant-level characteristics. Activity tracker data was evaluated for meeting quality standards $[6,7]$. Missing values and extreme values determined to be outliers (below the 0.05th percentile or above 0.95th percentile) were excluded from analysis or truncated. All analyses were conducted in R v.3.6.0 or higher.

\section{RESULTS}

\section{Study Participation}

Of the 2157 potential participants who were screened, 509 met the eligibility criteria, completed baseline assessments, connected their Fitbit to the study platform and were enrolled in the study (Fig. 1a). Of these, 350 participants had dense survey and activity data and were included in Analysis Population 1. A total of 283 participants had dense survey and heart rate data and were included in Analysis Population 2 (Fig. 1a).

\section{Baseline Demographics, Medical History, and Headache Characteristics}

Baseline demographics for Analysis Population $1 \quad(N=350)$ are presented in Table 1 . The majority of participants were female $(63.4 \%)$ and Caucasian/white $(81.7 \%)$ with a mean age of 36.1 (standard deviation [SD] 9.4) years. Over two-thirds of participants $(67.4 \%)$ had at least one self-reported comorbidity from a pre-selected list, the most common of which were allergies $(41.1 \%)$, anxiety (30.9\%), and depression $(20.9 \%) ; 66.3 \%$ reported a family history of headache (mother: 49.1\%; father: 16.3\%; sibling: 26.6\%). Migraineurs tended to have higher body mass index (BMI) and were more commonly female compared with TTH sufferers; no other differences were detected between headache types.

According to the questions adapted from the ICHD-3 classification guidelines, for Analysis Population 1, 60.9\% (213/350) of participants would be classified as having migraine or probable migraine, $31.1 \%(109 / 350)$ as experiencing TTH or probable TTH, and $0.6 \%(2 / 350)$ as experiencing cluster headache or probable cluster headache (Table 2). Due to the small sample size, participants with cluster headache or probable cluster headache were excluded from subsequent analysis. The average number of years since participants first experienced a headache was 17.8 (SD 10.6) years. The MIDAS score was 0-5 (no disability) for $14 \%$ of participants, 6-10 (mild disability) for $14.6 \%$ of 
Table 1 Baseline demographics

\begin{tabular}{|c|c|c|c|}
\hline Baseline demographics and medical history & $\begin{array}{l}\text { Analysis Population } 1 \\
(N=350)\end{array}$ & $\begin{array}{l}\text { TTH } \\
(N=109)\end{array}$ & $\begin{array}{l}\text { Migraine } \\
(N=213)\end{array}$ \\
\hline \multicolumn{4}{|l|}{ Age, years } \\
\hline Mean (SD) & $36.1(9.4)$ & $36.4(10.3)$ & $36.3(9.0)$ \\
\hline Median (IQR) & $34(11.0)$ & $34(13.0)$ & $35(11.0)$ \\
\hline Min; $\max$ & $18 ; 75$ & $18 ; 75$ & $22 ; 73$ \\
\hline \multicolumn{4}{|l|}{ Gender, $n(\%)$} \\
\hline Female & $222(63.4)$ & $47(43.1)$ & $158(74.2)$ \\
\hline Male & $127(36.3)$ & $62(56.9)$ & $54(25.4)$ \\
\hline Non-binary & $1(0.3)$ & $0(0)$ & $1(0.5)$ \\
\hline \multicolumn{4}{|l|}{ BMI } \\
\hline Mean (SD) & $29.2(6.3)$ & $28.2(5.8)$ & $30(6.6)$ \\
\hline Median (IQR) & $28.0(8.9)$ & $26.9(7.1)$ & $29.0(9.2)$ \\
\hline Min; $\max$ & $16.7 ; 47.4$ & $18.5 ; 47.1$ & $16.7 ; 47.4$ \\
\hline \multicolumn{4}{|l|}{ BMI categories, $\mathrm{kg} / \mathrm{m}^{2}$} \\
\hline Underweight: $<18.5$ & $5(1.4)$ & $1(0.9)$ & $3(1.4)$ \\
\hline Normal weight: $18.5-24.9$ & $94(26.9)$ & $35(32.1)$ & $48(22.5)$ \\
\hline Overweight: $25-29.9$ & $115(32.9)$ & $41(37.6)$ & $65(30.5)$ \\
\hline Obese: $\geq 30$ & $136(38.9)$ & $32(29.4)$ & $97(45.5)$ \\
\hline \multicolumn{4}{|l|}{ Ethnicity, $n(\%)$} \\
\hline Not hispanic or Latino & $317(90.6)$ & $100(91.7)$ & $191(89.7)$ \\
\hline Hispanic or Latino & $33(9.4)$ & $9(8.3)$ & $22(10.3)$ \\
\hline \multicolumn{4}{|l|}{ Race $^{\mathrm{a}}, n(\%)$} \\
\hline African-American/Black & $37(10.6)$ & $12(11.0)$ & $22(10.3)$ \\
\hline American Indian or Alaskan Native & $9(2.6)$ & $3(2.8)$ & $6(2.8)$ \\
\hline Asian & $25(7.1)$ & $10(9.2)$ & $12(5.6)$ \\
\hline Caucasian/white & $286(81.7)$ & $87(79.8)$ & $178(83.6)$ \\
\hline Other & $10(2.9)$ & $4(3.7)$ & $5(2.3)$ \\
\hline \multicolumn{4}{|l|}{ Highest level of education, $n$ (\%) } \\
\hline Did not complete high school & $1(0.3)$ & $0(0)$ & $1(0.5)$ \\
\hline High school graduate or GED & $23(6.6)$ & $5(4.7)$ & $17(8.0)$ \\
\hline Trade/technical/vocational training & $13(3.7)$ & $4(3.7)$ & $9(4.2)$ \\
\hline Some college, no degree & $49(14.0)$ & $13(12.1)$ & $29(13.6)$ \\
\hline
\end{tabular}


Table 1 continued

\begin{tabular}{llll}
\hline Baseline demographics and medical history & $\begin{array}{l}\text { Analysis Population } \mathbf{1} \\
(\boldsymbol{N}=\mathbf{3 5 0})\end{array}$ & $\begin{array}{l}\text { TTH } \\
(\boldsymbol{N}=\mathbf{1 0 9})\end{array}$ & $\begin{array}{l}\text { Migraine } \\
(\boldsymbol{N}=\mathbf{2 1 3})\end{array}$ \\
\hline $\begin{array}{l}\text { College graduate, associates or Bachelor of } \\
\text { Arts }\end{array}$ & $184(52.6)$ & $55(51.4)$ & $117(54.9)$ \\
Graduate degree & $65(18.6)$ & $26(24.3)$ & $31(14.6)$ \\
Doctorate degree & $13(3.7)$ & $4(3.7)$ & $9(4.2)$ \\
Missing & $2(0.6)$ & $2(1.8)$ & $0(0)$ \\
\hline
\end{tabular}

BMI Body mass index, GED General Education Development credential, IQR interquartile range, Min; max minimum; maximum, $S D$ standard deviation, $T T H$ tension-type headache

${ }^{\text {a }}$ Percentages may not add up to $100 \%$ since participants could select multiple answers

participants, 11-20 (moderate disability) for $25.7 \%$ of participants, and $\geq 21$ (severe) for $45.7 \%$ of participants in Analysis Population 1 (Table 2). When stratified by headache type, migraine sufferers experienced moderate/severe disability $(77.4 \%)$ more frequently than TTH sufferers (55.1\%), according to their MIDAS scores (Table 2). Participants reported multiple headache triggers using the following categories: sleep (e.g., fatigue/exhaustion), physiologic (e.g., physical activity), environmental (e.g., weather), exposure (e.g., smoking, alcohol), and other (e.g., stress). The most commonly reported headache triggers were physiologic (86.3\%) and sleep (83.1\%) (Table 2).

Baseline demographics, headache characteristics, and described triggers were similar in Analysis Population 2 (data not shown).

\section{Headache Descriptions from Daily Surveys}

During the 12-week study period-84 days across 350 participants $(29,400$ data entries)$83.4 \%$ of participants completed $75 \%$ of daily surveys, and $56 \%$ completed $90 \%$ of surveys. In $25.7 \%$ of surveys, participants described the previous day as a headache day. On days with headache, participants reported a headache intensity of 1-3 on 3297 days, intensity of 4-7 on 3867 days, and intensity of $8-10$ on 378 days. On days with headache, participants reported that the headache had no impact on $10.4 \%$ of days, a little impact on $51.2 \%$ of days, moderate impact on $27.3 \%$ of days, a lot of impact on $8.8 \%$ of days, and a great deal of impact on $2 \%$ of days (Table 3). Participants in Analysis Population 1 rated their health as very poor on $1.2 \%$ of days with headache, poor on $8.4 \%$ of days, fair on $42.3 \%$ of days, good on $42.2 \%$ of days, and very good on $5.7 \%$ of days with headache (Table 3 ).

\section{Associations Between Headache and Tracker-Based Activity}

Daily physical activity, sleep, and heart rate activity features that, after adjustment for age and gender, were statistically significantly associated with the occurrence of headache are summarized in Table 4 . On days when a headache occurred, participants in Analysis Population 1 were more likely to nap (OR $1.35 ; 95 \%$ CI $1.23,1.48 ; q<0.001)$ and to sleep more hours ( $\beta=0.07 ; 95 \%$ CI 0.03, 0.11; $q=0.002)$. Participants were on average less active on days with headache than on days without headache (19.9\% of the day spent inactive vs. $20.4 \%$ of the day, respectively; $q<0.001$ ) and took fewer steps (9413 vs. 9656 steps, respectively; $q<0.001)$.

Finally, days with headache were associated with lower maximum heart rate for the day $(\beta$ : $-0.45 ; 95 \%$ CI $-0.71,-0.19 ; q=0.002)$ and a lower maximum heart rate in any 5 -min period $(\beta:-0.64 ; 95 \%$ CI $-1.17,-0.12 ; q=0.037)$ (Table 4). 
Table 2 Baseline headache characteristics

\begin{tabular}{|c|c|c|c|}
\hline Characteristic & $\begin{array}{l}\text { Analysis Population } 1 \\
(N=350)\end{array}$ & $\begin{array}{l}\text { TTH } \\
(N=109)\end{array}$ & $\begin{array}{l}\text { Migraine } \\
(N=213)\end{array}$ \\
\hline \multicolumn{4}{|c|}{ Years since first experienced headache } \\
\hline Median (IQR) & $17.0(15.0)$ & $14.0(14.0)$ & $19.0(12.0)$ \\
\hline Min; $\max$ & $1 ; 50$ & $1 ; 45$ & $1 ; 50$ \\
\hline \multicolumn{4}{|c|}{ Headache type based on modified ICHD-3 Guidelines, $n$ (\%) } \\
\hline Migraine & $104(29.7)$ & - & $104(48.8)$ \\
\hline TTH & $56(16.0)$ & $56(51.4)$ & - \\
\hline Cluster headache & $2(0.6)$ & - & - \\
\hline Probable migraine $^{\mathrm{a}}$ & $109(31.1)$ & - & $109(51.2)$ \\
\hline Probable $\mathrm{TTH}^{\mathrm{a}}$ & $53(15.1)$ & $53(48.6)$ & - \\
\hline Probable cluster headache ${ }^{a}$ & $0(0)$ & - & - \\
\hline No classification ${ }^{\mathrm{b}}$ & $26(7.4)$ & - & - \\
\hline \multicolumn{4}{|c|}{ Headache-specific QoL: MIDAS score ${ }^{c}$} \\
\hline Median (IQR) & $18.0(25.8)$ & $11.0(19.0)$ & $22.0(29.0)$ \\
\hline Min; $\max$ & $0 ; 251$ & $0 ; 93$ & $0 ; 251$ \\
\hline \multicolumn{4}{|c|}{ Headache-specific QoL: MIDAS categories/scores, $n$ (\%) } \\
\hline No disability $(0-5)$ & $49(14.0)$ & $25(22.9)$ & $22(10.3)$ \\
\hline Mild disability $(6-10)$ & $51(14.6)$ & $24(22.0)$ & $26(12.2)$ \\
\hline Moderate disability (11-20) & $90(25.7)$ & $26(23.9)$ & $51(23.9)$ \\
\hline Severe disability $(\geq 21)$ & $160(45.7)$ & $34(31.2)$ & $114(53.5)$ \\
\hline \multicolumn{4}{|l|}{ Headache triggers ${ }^{\mathrm{d}}, n(\%)$} \\
\hline Sleep & $291(83.1)$ & $91(83.5)$ & $177(83.1)$ \\
\hline Physiologic $^{\mathrm{e}}$ & $302(86.3)$ & $89(81.7)$ & $187(87.8)$ \\
\hline Environmental & $101(28.9)$ & $22(20.2)$ & $73(34.3)$ \\
\hline Exposure & $252(72.0)$ & $70(64.2)$ & $160(75.1)$ \\
\hline Other & $29(8.3)$ & $9(8.3)$ & $18(8.5)$ \\
\hline
\end{tabular}


Table 2 continued

\begin{tabular}{llll}
\hline Characteristic & $\begin{array}{l}\text { Analysis Population 1 } \\
(\boldsymbol{N}=350)\end{array}$ & $\begin{array}{l}\text { TTH } \\
(\boldsymbol{N}=\mathbf{1 0 9})\end{array}$ & $\begin{array}{l}\text { Migraine } \\
(\boldsymbol{N}=\mathbf{2 1 3})\end{array}$ \\
\hline None of the above & $2(0.6)$ & $0(0)$ & $2(0.9)$ \\
\hline
\end{tabular}

$I C H D-3$ international classification of headache disorders, IQR interquartile range, MIDAS Migraine Disability Assessment Questionnaire, $Q o L$ quality of life, $S D$ standard deviation, $T T H$ tension-type headache

aParticipants who were not classified with a headache type using ICHD-3 guidelines may be classified according to 'probable' type. Participants who fulfilled all but one criterion required for classification were classified as a 'probable' headache type. Per guidelines (section Data Sources and Variables), if a participant was both 'probable migraine' and 'probable TTH,' they were classified as 'probable migraine.' 'Probable cluster headache' was assessed if there were no other probable classifications barticipants with a headache that could not be classified using the ICHD-3 Guidelines based on their self-reported symptoms

${ }^{c}$ Participants reported the number of days their migraine affected each of 5 activities, from 0 to 90 days. The MIDAS score is calculated as a sum of these days, ranging from 0 to 450 days (i.e., $90 \times 5$ ), where higher sums indicate greater disability. The sum score is then categorized using the 4 recommended buckets: $0-5$ days = no disability; $6-10$ days = mild disability; $11-20$ days $=$ moderate disability; and $\geq 21$ days = severe disability

${ }^{\mathrm{d}}$ Percentages may not add up to $100 \%$ since participants could select multiple answers

${ }^{\text {e}}$ Physiologic health triggers included exercise or physical activity, eye strain, dry eyes, and menstruation

\section{Association Between Impact of Headache on Daily Life and Tracker-Based Activity}

Days with headache causing greater impact on daily life were associated with higher odds of napping (on headache days with a great deal of impact vs. days with no impact, the OR for napping was $4.87 ; 95 \%$ CI 2.89, 8.19, $q<0.001)$. Participants also slept fewer hours the previous night ('last night') $(B:-0.07$; CI $0.12,-0.02 ; q=0.017)$; on days with headache causing no impact, participants slept $6.9 \mathrm{~h}$ on average while on days with headaches causing a great deal of impact, participants slept $6.5 \mathrm{~h}$ on average (Table 5). Participants also tended to spend a greater fraction of sleep time awake $(\beta$ : 0.22 ) as the headache impact increased. On days with headache with greater impact, participants were less active (9685 steps on days with 'no impact' of headache vs. 7966 steps on days with 'a great deal of impact' of headache). Similarly, both the maximum heart rate and maximum heart rate during any 5-min period were negatively associated with headachespecific impact, whereas other heart rate variables did not show a statistically significant association (Table 5).

\section{Association Between Impact of Headache on Self-Rated Health and Tracker-Based Activity}

Participants recorded their self-rated health as 'fair' and as 'good' on 42.3 and $42.2 \%$ of all evaluable days with headache, respectively. Compared to headache-related impact on QoL, inverse relationships between self-rated health and activity were found (Table 6). Days with higher self-rated health were associated with lower odds of taking a nap (on days with very good health vs. days with very poor health, the OR for napping was 0.14; CI 0.07, 0.28; $q<0.001)$ and less time spent awake during sleep the previous night $(\beta:-1.28$; CI -2.23 , $0.33 ; q=0.030)$. On average, participants took 6586 steps on days self-rated as 'very poor' in health while participants took 11,266 steps on average on days self-rated as 'very good health

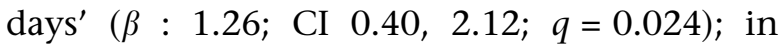
parallel, more time was spent being active $(\beta$ : 2.29; CI 0.97, 3.62; $q=0.011$ ).

\section{Associations Between Headache and Tracker-Based Activity by Headache Type}

In the analysis stratified by headache type (migraine or TTH), significant associations between 
Table 3 Summary of headache descriptions from daily surveys-Analysis Population 1

\begin{tabular}{|c|c|c|c|}
\hline Headache descriptions & $\begin{array}{l}\text { Analysis Population } 1 \\
(N=350)\end{array}$ & $\begin{array}{l}\text { TTH } \\
(N=109)\end{array}$ & $\begin{array}{l}\text { Migraine } \\
(N=213)\end{array}$ \\
\hline $\mathrm{N}_{\text {days }}$ total & 29,400 & 9156 & 17,892 \\
\hline $\mathrm{N}_{\text {days }}$ per participant, mean $(\mathrm{SD})$ & $73.3(11.6)$ & $72.6(11.3)$ & $73.7(11.4)$ \\
\hline \multicolumn{4}{|l|}{ Day with headache, $N_{\text {days }}(\%)$} \\
\hline Headache & $7542(25.7)$ & $2164(23.6)$ & $4687(26.2)$ \\
\hline No headache & $18,097(61.6)$ & $5746(62.8)$ & $11,011(61.5)$ \\
\hline Missing & $3761(12.8)$ & $1246(13.6)$ & $2194(12.3)$ \\
\hline \multicolumn{4}{|l|}{ Headache Intensity } \\
\hline Median (IQR) & $4(2)$ & $3(3)$ & $4(3)$ \\
\hline Min; $\max$ & $1 ; 10$ & $1 ; 9$ & $1 ; 10$ \\
\hline \multicolumn{4}{|l|}{ Life impact ${ }^{\mathrm{a}}$} \\
\hline Median (IQR) & $2(1)$ & $2(1)$ & $2(1)$ \\
\hline Min; $\max$ & $1 ; 5$ & $1 ; 5$ & $1 ; 5$ \\
\hline \multicolumn{4}{|l|}{ Life impact ${ }^{\mathrm{a}}, n(\%)$} \\
\hline No impact at all & $783(10.4)$ & $307(14.2)$ & $413(8.8)$ \\
\hline A little impact & $3865(51.2)$ & $1193(55.1)$ & $2338(49.9)$ \\
\hline A moderate impact & $2056(27.3)$ & $536(24.8)$ & $1291(27.5)$ \\
\hline A lot of impact & $663(8.8)$ & $108(5.0)$ & $496(10.6)$ \\
\hline A great deal of impact & $149(2.0)$ & $12(0.6)$ & $133(2.8)$ \\
\hline Missing & $26(0.3)$ & $8(0.4)$ & $16(0.3)$ \\
\hline \multicolumn{4}{|l|}{ Daily self-rated health ${ }^{\mathrm{a}}$} \\
\hline Median (IQR) & $3(1)$ & $3(1)$ & $3(1)$ \\
\hline Min; $\max$ & $1 ; 5$ & $1 ; 5$ & $1 ; 5$ \\
\hline \multicolumn{4}{|l|}{ Daily self-rated health ${ }^{\mathrm{a}}, n(\%)$} \\
\hline Very poor & $87(1.2)$ & $17(0.8)$ & $66(1.4)$ \\
\hline Poor & $630(8.4)$ & $154(7.1)$ & $421(9.0)$ \\
\hline Fair & $3191(42.3)$ & $973(45.0)$ & $1978(42.2)$ \\
\hline Good & $3179(42.2)$ & $862(39.8)$ & $1976(42.2)$ \\
\hline Very good & $429(5.7)$ & $150(6.9)$ & $230(4.9)$ \\
\hline Missing & $26(0.3)$ & $8(0.4)$ & $16(0.3)$ \\
\hline
\end{tabular}

$I Q R$ interquartile range; $S D$ standard deviation; $T T H$ tension-type headache ${ }^{a}$ Assessed on headache days only 
Table 4 Associations between headache and tracker-based activity features (activity, sleep and heart rate)-Analysis Population 1

\begin{tabular}{lcr}
\hline Activity features & $\mathbf{O R}^{\mathbf{a}}(\mathbf{9 5} \% \mathbf{C I})$ & $\boldsymbol{q}$ \\
\hline Nap taken & $1.35(1.23,1.48)$ & $\boldsymbol{q} 0.001$ \\
\hline & $\boldsymbol{\beta}^{\mathbf{a}}(\mathbf{9 5 \%} \mathbf{C I})$ & 0.558 \\
\hline Total hours of sleep last night (h) & $0.01(-0.03,0.06)$ & 0.411 \\
Fraction of time spent awake last night (\%) & $0.06(-0.06,0.17)$ & $<0.001$ \\
Total number of steps in a day (1000 steps) & $-0.24(-0.35,-0.13)$ & $<0.001$ \\
Proportion of day with non-zero steps (\%) & $-0.43(-0.59,-0.26)$ & 0.464 \\
Time of maximum number of steps in a 30-min period (h) & $0.05(-0.07,0.18)$ & 0.029 \\
Maximum number of steps in a 30-min period (1000 steps) & $-0.03(-0.06,-0.01)$ & 0.002 \\
Total hours of sleep (h) & $0.07(0.03,0.11)$ & 0.237 \\
Fraction of time spent awake (\%) & $0.09(-0.03,0.20)$ & 0.073 \\
Number of times woken up & $0.11(0.01,0.22)$ & 0.002 \\
Maximum daily heart rate (bpm) & $-0.45(-0.71,-0.19)$ & 0.385 \\
Resting heart rate (bpm) & $0.05(-0.04,0.13)$ & 0.037 \\
Maximum heart rate in a 5-min period (bpm) & $-0.64(-1.17,-0.12)$ & 0.363 \\
Proportion of day spent inactive (\%) & $0.18(-0.12,0.48)$ & \\
\hline Regress
\end{tabular}

$\beta$ Regression coefficient, $b p m$ beats per minute, $C I$ confidence interval, $O R$ odds ratio, $q P$ values with Benjamini-Hochberg correction for multiple testing

${ }^{a}$ Adjusted coefficients were derived from regression models with day with headache as independent variable and activity feature as dependent variable

days with headache and duration of sleep, physical activity, and heart rate were found in the subgroup of participants suffering from migraine, but these associations were not found in the subgroup of participants suffering from TTH (Fig. 2). Significant associations were found in both subgroups between headache-related impact on QoL and various sleep, activity, and heart rate features (directions were similar to those summarized above). Interestingly, the pattern of associations was different for selfrated health, where significant positive associations were found with activity (steps) only for those with migraine (Fig. 2).

\section{Treatment Preferences and Decisions}

The most common baseline treatment for headache symptoms was over-the-counter (OTC) pain relievers (used by $98.3 \%$ of participants at least once), and medication choice was commonly influenced by symptom severity, duration and prior personal experience with the medication (Electronic Supplementary Material [ESM] Table 1). Two-fifths of participants $(38.9 \%)$ reported using non-medication treatment strategies when medications were unsuccessful. The majority of participants chose relaxation techniques (58.6\%), hot/cold compress $(55.4 \%)$, and improve of sleep habits $(50.9 \%)$ as alternative strategies for headache relief. 
Table 5 Associations between impact of headache on QoL and activity variables (sleep, steps, heart rate)-Analysis Population 1

\begin{tabular}{|c|c|c|c|c|c|}
\hline Activity variables & $\mathbf{O R}^{\mathrm{a}}$ & $95 \% \mathrm{CI}$ & $\begin{array}{l}\text { Proportion (\%) } \\
\text { of 'No impact' } \\
\text { days with nap(s) }\end{array}$ & $\begin{array}{l}\text { Proportion (\%) of 'A } \\
\text { great deal of impact' } \\
\text { days with nap(s) }\end{array}$ & $q$ \\
\hline Nap taken (little impact vs. no impact) & 1.16 & $0.88,1.53$ & $7.39 \%$ & $27.99 \%$ & 0.289 \\
\hline $\begin{array}{l}\text { Nap taken (moderate impact vs. no } \\
\text { impact) }\end{array}$ & 1.53 & $1.14,2.07$ & & & 0.004 \\
\hline $\begin{array}{l}\text { Nap taken (a lot of impact vs. no } \\
\text { impact) }\end{array}$ & 2.51 & $1.76,3.57$ & & & $<0.001$ \\
\hline $\begin{array}{l}\text { Nap taken (a great deal of impact vs. no } \\
\text { impact) }\end{array}$ & 4.87 & $2.89,8.19$ & & & $<0.001$ \\
\hline Activity variables & $\beta^{a}$ & $95 \% \mathrm{CI}$ & $\begin{array}{l}\text { Mean on } \\
\text { 'No impact' } \\
\text { days }\end{array}$ & $\begin{array}{l}\text { Mean on 'A great } \\
\text { deal of impact' } \\
\text { days }\end{array}$ & $q$ \\
\hline Total hours of sleep last night (h) & -0.07 & $-0.12,-0.02$ & 6.86 & 6.47 & 0.017 \\
\hline $\begin{array}{l}\text { Fraction of time spent awake last night } \\
\text { (\%) }\end{array}$ & 0.22 & $0.09,0.35$ & 7.49 & 9.24 & 0.002 \\
\hline $\begin{array}{l}\text { Total number of steps in a day (1000 } \\
\text { steps) }\end{array}$ & -0.41 & $-0.53,-0.30$ & 9.69 & 7.97 & $<0.001$ \\
\hline $\begin{array}{l}\text { Proportion of day with non-zero steps } \\
\text { (\%) }\end{array}$ & -0.71 & $-0.89,-0.53$ & 20.49 & 17.26 & $<0.001$ \\
\hline $\begin{array}{l}\text { Time of maximum number of steps in a } \\
30-\min \text { period }(\mathrm{h})\end{array}$ & -0.12 & $-0.25,0.01$ & 14.66 & 13.99 & 0.115 \\
\hline $\begin{array}{l}\text { Maximum number of steps in a } 30-\mathrm{min} \\
\text { period (1000 steps) }\end{array}$ & -0.07 & $-0.10,-0.05$ & 1.71 & 1.36 & $<0.001$ \\
\hline Total hours of sleep (h) & -0.03 & $-0.08,0.02$ & 6.94 & 6.86 & 0.255 \\
\hline Fraction of time spent awake (\%) & 0.19 & $0.06,0.31$ & 7.38 & 8.57 & 0.009 \\
\hline Number of times woken up & 0.05 & $-0.07,0.17$ & 8.60 & 8.94 & 0.460 \\
\hline Maximum daily heart rate (bpm) & -0.54 & $-0.82,-0.26$ & 102.47 & 100.29 & $<0.001$ \\
\hline Resting heart rate (bpm) & 0.05 & $-0.05,0.15$ & 66.25 & 66.36 & 0.329 \\
\hline $\begin{array}{l}\text { Maximum heart rate in a 5-min period } \\
(\mathrm{bpm})\end{array}$ & -1.13 & $-1.69,-0.57$ & 124.65 & 119.47 & $<0.001$ \\
\hline Proportion of day spent inactive (\%) & 0.11 & $-0.22,0.44$ & 75.09 & 75.41 & 0.528 \\
\hline
\end{tabular}

Activity features were modeled as continuous outcomes, with life impact as the predictor and with age and sex as covariates, taking intra-individual correlation into account via mixed-effects modeling

$\beta$ regression coefficient, $C I$ confidence interval, $O R$ odds ratio, $q P$-values with Benjamini -Hochberg correction for multiple testing

${ }^{a}$ Adjusted coefficients were derived from mixed-effects models using age and gender as covariates 
Table 6 Associations between self-reported health and activity variables (sleep, steps, heart rate)-Analysis Population 1

\begin{tabular}{|c|c|c|c|c|c|}
\hline Activity variables & $\mathbf{O R}^{\mathbf{a}}$ & $95 \% \mathrm{CI}$ & $\begin{array}{l}\text { Proportion (\%) } \\
\text { of 'Very poor } \\
\text { health' } \\
\text { days nap was } \\
\text { taken }\end{array}$ & $\begin{array}{l}\text { Proportion (\%) } \\
\text { of 'Very } \\
\text { good health' days } \\
\text { nap } \\
\text { was taken }\end{array}$ & $q$ \\
\hline Nap taken (poor health vs. very poor health) & 0.68 & $0.38,1.21$ & $25.5 \%$ & $4.5 \%$ & 0.181 \\
\hline Nap taken (fair health vs. very poor health) & 0.39 & $0.22,0.68$ & & & $<0.001$ \\
\hline Nap taken (good health vs. very poor health) & 0.23 & $0.13,0.41$ & & & $<0.001$ \\
\hline $\begin{array}{l}\text { Nap taken (very good health vs. very poor } \\
\text { health) }\end{array}$ & 0.14 & $0.07,0.28$ & & & $<0.001$ \\
\hline Activity variables & $\beta^{\mathbf{a}}$ & $95 \% \mathrm{CI}$ & $\begin{array}{l}\text { Mean on } \\
\text { 'Very poor } \\
\text { health' days }\end{array}$ & $\begin{array}{l}\text { Mean on } \\
\text { 'Very good } \\
\text { health' days }\end{array}$ & $q$ \\
\hline Total hours of sleep last night $(\mathrm{h})$ & 0.06 & $-0.31,0.44$ & 6.47 & 6.95 & 0.840 \\
\hline Fraction of time spent awake last night (\%) & -1.28 & $-2.23,-0.33$ & 9.13 & 7.19 & 0.030 \\
\hline Total number of steps in a day (1000 steps) & 1.26 & $0.40,2.12$ & 6.59 & 11.27 & 0.024 \\
\hline Proportion of day with non-zero steps (\%) & 2.29 & $0.97,3.62$ & 15.47 & 22.21 & 0.011 \\
\hline $\begin{array}{l}\text { Time of maximum number of steps in a } \\
30-\min \text { period }(\mathrm{h})\end{array}$ & 1.42 & $0.42,2.43$ & 13.06 & 14.78 & 0.025 \\
\hline $\begin{array}{l}\text { Maximum number of steps in a } 30-\mathrm{min} \\
\text { period (1000 steps) }\end{array}$ & 0.15 & $-0.05,0.34$ & 1.20 & 2.11 & 0.267 \\
\hline Fraction of time spent awake (\%) & -0.26 & $-1.20,0.69$ & 8.36 & 7.01 & 0.778 \\
\hline Number of times woken up & -0.92 & $-1.82,-0.03$ & 9.74 & 8.37 & 0.126 \\
\hline Maximum daily heart rate (bpm) & -0.21 & $-2.30,1.87$ & 101.57 & 105.44 & 0.890 \\
\hline Resting heart rate (bpm) & 0.19 & $-0.52,0.90$ & 66.78 & 65.73 & 0.778 \\
\hline Proportion of day spent inactive (\%) & -1.90 & $-4.34,0.55$ & 77.80 & 74.25 & 0.257 \\
\hline
\end{tabular}

Activity features were modeled as continuous outcomes, with self-reported health as the predictor and with age and sex as covariates, taking intra-individual correlation into account via mixed-effects modeling

$\beta$ regression coefficient, $C I$ confidence interval, $O R$ odds ratio, $q$ P-values with Benjamini -Hochberg correction for multiple testing

${ }^{a}$ Adjusted coefficients were derived from mixed-effects models using age and gender as covariates

During the study, participants took medication for headache for a median of $55.2 \%$ (interquartile range [IQR] $47.3 \%$ ) of all days with headache, and used a non-medication strategy for a median of $45.5 \%$ (IQR $61.5 \%$ ) of all days with headache. The most common medication types used at least once were non-steroid anti- inflammatory drugs (NSAIDs) (both OTC and prescription; $79.9 \%$ ), OTC non-NSAID pain relievers $(68.2 \%)$, and prescription preventive medication (32.4\%). Participants reported taking medication when bothered by headache or to prevent their headache from getting worse; the most common reason for not taking 


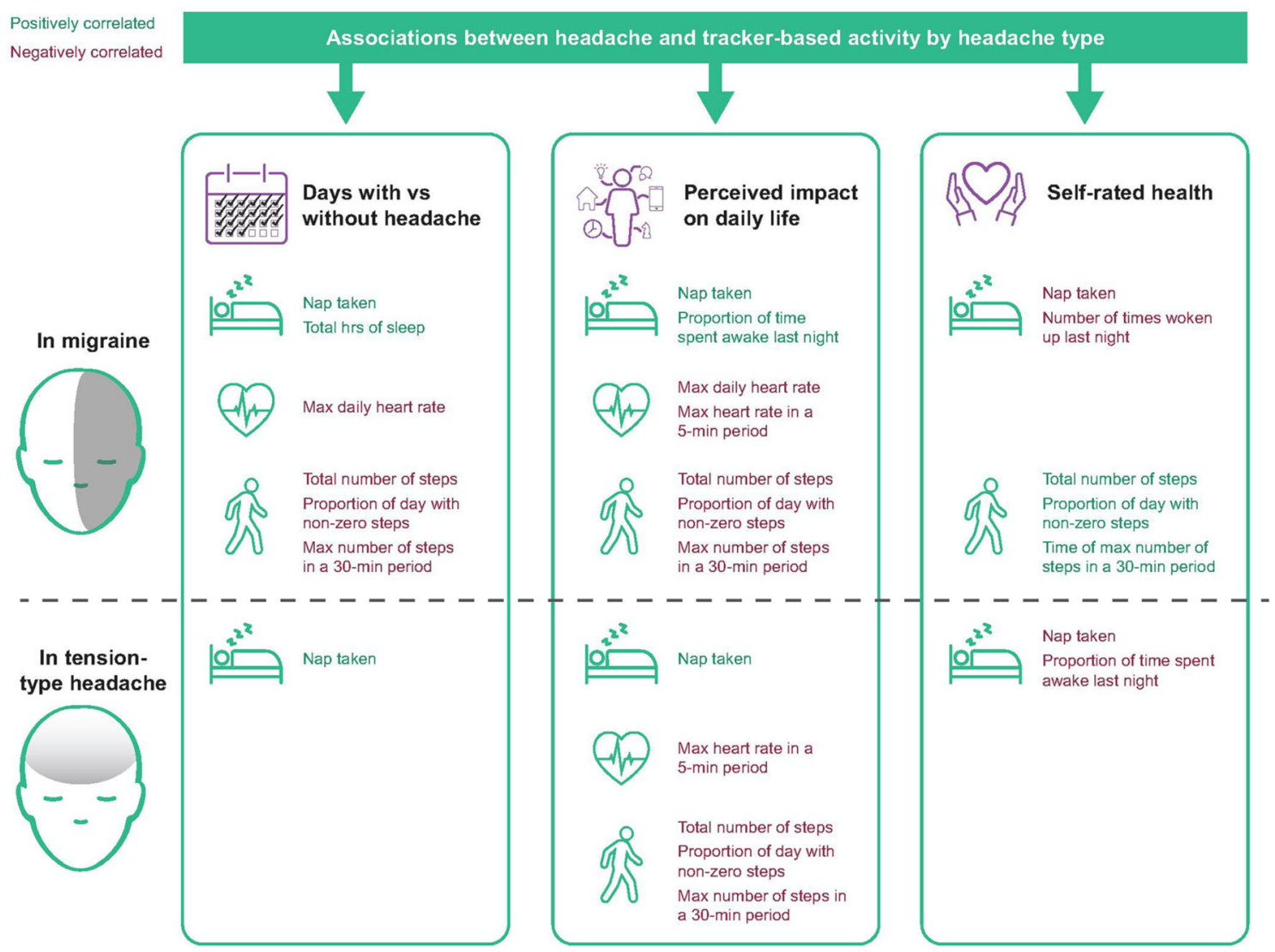

Fig. 2 Summary of statistically significant associations between activity variables, days with headache, and quality of life in participants stratified by headache type-Analysis Population 1

medication on a day with headache was that 'headache pain was tolerable' (ESM Table 2). There were no differences between users of prescription medication and participants who did not use it in terms of demographics, comorbidities, headache type and intensity, or tracker-based activity levels (data not shown).

The results presented above were obtained on Analysis Population 1, which included participants with dense data from daily surveys and activity trackers. The results obtained for Analysis Population 2 (participants with dense heart rate data) were broadly similar (data not shown).

\section{DISCUSSION}

The aims of this study were to analyze the impact of headache on passively collected tracker-based activity variables, to explore their associations with QoL in individuals suffering from frequent episodic primary headache, and to understand headache-related treatment preferences and decisions. The data analysis captured headache burden across multiple dimensions, including occurrence, pain intensity, treatment choices, and perceived impact on daily life and self-rated health.

Our analysis showed that a number of tracker-based activity features were consistently associated with headache burden. Overall, compared with days without headache, days with headache were associated with reduced daily step count, decreased proportion of the day spent active, and increased daytime napping. With respect to heart rate, we showed that maximum heart rate was lower on days with headache, which may indicate lower levels of physical activity. Notably, when analyses were stratified by headache type, daily step count 
appeared to be a particularly consistent indicator of headache burden among migraine sufferers (but not TTH sufferers), as it was consistently associated with all three self-reported measures: headache occurrence, impact on daily life, and self-rated health. These particular data streams may serve as candidates for further validation work and future consideration regarding clinical relevance.

These findings are consistent with previous research indicating that migraine sufferers may experience more headache burden than TTH sufferers $[8,9]$. Previous studies also showed that the overall perception of QoL, health, and physical activity were significantly lower among migraine sufferers compared with other headache types, emphasizing that migraine burden is, in general, underestimated and poorly managed [8-13]. These studies highlight the lack of research focusing on 'common headache' versus plethoric data available for migraine sufferers. For example, the relationship between migraines, self-reported QoL measures and behaviors such as physical activity and sleep, have been investigated in several large population-based studies; however, fewer studies have investigated the relationship between these measures and other headache types, such as TTH $[1,3,14,15]$. This study adds support and expands the existing knowledge of the links between physical activity and both migraine and TTH.

The recent widespread use of consumer physical activity trackers offers unprecedented opportunities to gather vital real-world data, with many studies already under way (one review stated that 127 studies were registered on ClinicalTrials.gov as of 11 November 2006) [16]. Fitbits have been deemed useful for measuring general activity levels in real-world settings, having demonstrated acceptable reliability and high sensitivity; a study comparing ten different activity trackers found that Fitbit outperforms many comparators in terms of tracking step counts $[17,18]$.

To our knowledge, this study is one of the first to analyze Fitbit data in association with headache. The particular strengths of this study stem from its practical design, the use of passive data collection from activity trackers, the inclusion of headache sufferers from the wider community, i.e., those who do not usually seek treatment in primary care, and analysis of daily diaries of participant-reported outcomes. The prospectively collected data from this study provide a more detailed picture of the everyday life of individuals with headache and allows researchers to generate additional hypotheses on the timing and context of headache episodes.

\section{Limitations}

Alongside the strengths of this study, some limitations warrant discussion. Headache classification was self-reported, not diagnosed by a professional, and, therefore, is prone to misclassification. For example, in this study, the ability of participants to classify their headache type correctly compared with ICHD-3 guidelines was poor (analysis not shown). However, the classification of headache type used in our analysis was based on self-reported symptoms and ICHD-3 guidelines rather than perceived classification, which helped to address this potential limitation. Another limitation was that the majority of participants identified as female and Caucasian; $74.9 \%$ had a college degree or higher education, compared with $61.1 \%$ overall in the USA [19]. Further, this population was also more active overall, averaging a daily step count of approximately $8000 /$ day, compared with the U.S. national average of 5000/day [20]. Taken together, the associations between activity and headache observed in this study may not generalize to more racially diverse and less active individuals. Furthermore, since the analysis focused on the subset of headache sufferers who were able to complete the majority of their daily surveys and regularly wear their Fitbit, data may be lacking for participants who experienced very severe headaches and were subsequently less compliant to study protocols. As a result, associations may not fully represent the experience of individuals with frequent headaches who do not regularly use tracking devices. Lastly, although this study focused on medication usage patterns in headache sufferers, further analysis would be 
needed to explore associations between medication use and behavioral features. Further analysis would also be needed to explore autonomic differences during different phases of headache and migraine, as heart rate variability data were not collected during this study.

Although real-world data cannot replace randomized controlled trials, they help to fill the 'efficacy-effectiveness gap', and can provide insights on how to improve headache sufferers' QoL [21]. Additionally, compared with the highly selective populations examined in clinical trials, real-world data allow for the examination of heterogeneous populations with varying comorbidities, often more comparable to the general population. As such, real-world data are increasingly being used to complement randomized controlled trials. Real-world data can examine aspects of headache burden and treatment preferences and decisions that are commonly overlooked, and inform clinical trial design and other aspects of drug development, providing an opportunity to accelerate and increase patient access to innovative medicines [22].

\section{CONCLUSION}

Taken together, the findings provide preliminary evidence that activity trackers are useful tools for quantifying headache burden among individuals with frequent headaches in realworld settings. The data obtained in this study can be used to help develop new metrics that can be used in future research, and to increase understanding of the diversity in self-treatment patterns outside primary care.

\section{ACKNOWLEDGEMENTS}

The authors are grateful to the study participants for their contribution to the study. The authors would like to thank Valérie Bulidon and Dorothea Greifenberg of Sanofi Consumer Healthcare for project management of scientific communications.
Funding. This study was sponsored by Sanofi. The Rapid Service Fee was funded by Sanofi.

Medical Writing and Editorial Assistance. Medical writing and editorial support was provided by Nichola Cruickshanks of inScience Communications, Springer Healthcare Ltd, UK, and was funded by Sanofi.

Authorship. All named authors meet the International Committee of Medical Journal Editors (ICMJE) criteria for authorship for this article, take responsibility for the integrity of the work as a whole, and have given their approval for this version to be published.

Authors' Contributions. Initial drafts of the introduction and discussion were developed by PJG with substantial edits by CJC; all other authors provided comments and feedback on later drafts.

Disclosures. Peter J Goadsby reports grants and personal fees from Amgen and Eli-Lilly and Company; a grant from Celgene; personal fees from Alder Biopharmaceuticals, Aeon Biopharma, Allergan, Biohaven Pharmaceuticals Inc., Electrocore LLC, eNeura, Epalex, Glaxosmithkline, Impel Neuropharma, Lundbeck, Novartis, Pfizer, Praxis, Sanofi, Santara Therapeutics, Satsuma, and Teva Pharmaceuticals; personal fees from MedicoLegal work, Massachusetts Medical Society, Up-to-Date, Oxford University Press, and Wolters Kluwer; and a patent magnetic stimulation for headache assigned to eNeura without fee. Luminita Constantin, Simon Hitier, Iva Igracki Turudic, Caroline Amand-Bourdon, Andrew Stewart and Caty Ebel-Bitoun are employees of Sanofi. Christian J Cerrada and Jae S Min are employees of Evidation Health.

Compliance with Ethics Guidelines. This study was performed in accordance with the Helsinki Declaration of 1964 and its later amendments. Signed informed consent was obtained electronically from participants prior to enrollment using Evidation Health's eConsent process. The study protocol, informed 
consent form, recruitment materials, and all participant materials were approved by the Western Institutional Review Board (Puyallup, WA, USA; WIRB protocol \#20190326).

Data Availability. The datasets generated during and/or analyzed during the current study are not publicly available as participant consent limits access to the study sponsor and Evidation Health.

Open Access. This article is licensed under a Creative Commons Attribution-NonCommercial 4.0 International License, which permits any non-commercial use, sharing, adaptation, distribution and reproduction in any medium or format, as long as you give appropriate credit to the original author(s) and the source, provide a link to the Creative Commons licence, and indicate if changes were made. The images or other third party material in this article are included in the article's Creative Commons licence, unless indicated otherwise in a credit line to the material. If material is not included in the article's Creative Commons licence and your intended use is not permitted by statutory regulation or exceeds the permitted use, you will need to obtain permission directly from the copyright holder. To view a copy of this licence, visit http:// creativecommons.org/licenses/by-nc/4.0/.

\section{REFERENCES}

1. Amin FM, Aristeidou S, Baraldi C, et al. The association between migraine and physical exercise. J Headache Pain. 2018;19:83.

2. Houle TT, Butschek RA, Turner DP, et al. Stress and sleep duration predict headache severity in chronic headache sufferers. Pain. 2012;153:2432-40.

3. Varkey E, Hagen K, Zwart JA, et al. Physical activity and headache: results from the Nord-Trondelag Health Study (HUNT). Cephalalgia. 2008;28: 1292-7.

4. Stewart WF, Lipton RB, Dowson AJ, et al. Development and testing of the Migraine Disability Assessment (MIDAS) questionnaire to assess headacherelated disability. Neurology. 2001;56:S20-28.
5. Headache Classification Committee of the International Headache Society (IHS). The International Classification of Headache Disorders, 3rd edition. Cephalalgia. 2018;38:1-211.

6. Tudor-Locke C, Camhi SM, Troiano RP. A catalog of rules, variables, and definitions applied to accelerometer data in the National Health and Nutrition Examination Survey, 2003-2006. Prev Chron Dis. 2012;9:E113.

7. Migueles JH, Cadenas-Sanchez C, Ekelund U, et al. Accelerometer data collection and processing criteria to assess physical activity and other outcomes: a systematic review and practical considerations. Sports Med. 2017;47:1821-45.

8. Shaik MM, Hassan NB, Tan HL, et al. Quality of life and migraine disability among female migraine patients in a tertiary hospital in Malaysia. Biomed Res Int. 2015;2015:523717. https://doi.org/10. $1155 / 2015 / 523717$.

9. Kroll LS, Hammarlund CS, Westergaard ML, et al. Level of physical activity, well-being, stress and selfrated health in persons with migraine and co-existing tension-type headache and neck pain. J Headache Pain. 2017;18:46.

10. Iigaya M, Sakai F, Kolodner KB, et al. Reliability and validity of the Japanese Migraine Disability Assessment (MIDAS) Questionnaire. Headache. 2003;43: 343-52.

11. Leonardi M, Raggi A, Bussone G, et al. Health-related quality of life, disability and severity of disease in patients with migraine attending to a specialty headache center. Headache. 2010;50:1576-86.

12. Michel P, Dartigues JF, Lindoulsi A, et al. Loss of productivity and quality of life in migraine sufferers among French workers: results from the GAZEL cohort. Headache. 1997;37:71-8.

13. Sharma K, Remanan R, Singh S. Quality of life and psychiatric comorbidity in Indian migraine patients: a headache clinic sample. Neurol India. 2013;61:355-9.

14. Ahn AH. Why does increased exercise decrease migraine? Curr Pain Headache Rep. 2013;17:379.

15. Fernandez-de-Las-Penas C, Fernandez-Munoz JJ, Palacios-Cena $\mathrm{M}$, et al. Sleep disturbances in tension-type headache and migraine. Ther Adv Neurol Disord. 2018;11:1756285617745444.

16. Wright SP, Hall Brown TS, Collier SR, et al. How consumer physical activity monitors could transform human physiology research. Am J Physiol Regul Integr Comp Physiol. 2017;312:R358-67. 
17. Bartlett SJ, Barnes $T$, McIvor RA. Integrating patients into meaningful real-world research. Ann Am Thorac Soc. 2014;11(Suppl 2):S112-7.

18. An HS, Jones GC, Kang SK, et al. How valid are wearable physical activity trackers for measuring steps? Eur J Sport Sci. 2017;17:360-8.

19. United States Census Bureau. Educational attainment in the United States. 2019. https://www. census.gov/data/tables/2019/demo/educationalattainment/cps-detailed-tables.html Accessed 29 June 2020.
20. Althoff T, Sosic R, Hicks JL, et al. Large-scale physical activity data reveal worldwide activity inequality. Nature. 2017;547:336-9.

21. Eichler HG, Abadie E, Breckenridge A, et al. Bridging the efficacy-effectiveness gap: a regulator's perspective on addressing variability of drug response. Nat Rev Drug Discov. 2011;10:495-506.

22. Makady A, de Boer A, Hillege $\mathrm{H}$, et al. What is realworld data? A review of definitions based on literature and stakeholder interviews. Value Health. 2017;20:858-65. 